\title{
Erratum to: Occlusion intestinale en zone d'endémie : penser aussi à la tuberculose
}

\section{Erratum to: Intestinal Obstruction in Endemic Areas: Don't Forget Digestive Tuberculosis}

M.M. Diop - V.M.P. Cisse $\cdot$ A. Berthé $\cdot$ J.M. Reimund $\cdot$ P.A. Ba $\cdot$ P.S. Touré $\cdot$ A.A. Dia $\cdot$ F.A. Faye $\cdot$ B.M. Diop $\cdot$ M.M. Ka

(C) Lavoisier SAS 2016

Erratum to : Acta Endosc. DOI 10.1007/s10190-015-0527-9

La liste des auteurs est incomplète, les auteurs sont : M.M. Diop, V.M.P. Cisse, A. Berthé, J.M. Reimund, P.A. Ba, P.S. Touré, A.A. Dia, F.A. Faye, B.M. Diop, M.M. Ka 